\title{
Infochemicals for the chromatographic separation technology
}

\author{
Haidi Li ${ }^{1, a}$, Weiping Yin ${ }^{* 1, b}$, Huaqing $\mathrm{Liu}^{1, \mathrm{c}}$ and Yanjun $\mathrm{Li}^{1, \mathrm{~d}}$ \\ ${ }^{1}$ Faculty of Chemical and Pharmaceutical Engineering, Henan University of Scientific and \\ Technology, Henan, Luoyang, 471023, China. \\ a1157382625@qq.com, ’byinwp@haust.edu.cn, ${ }^{c}$ liuhuaqing@163.com, \\ d1625043712@qq.com;
}

Keywords: Infochemicals, chromatography separation, NP-HPLC, Sephadex LH-20, secondary metabolites, Polyrhachis vicina Roger.

Abstract. Chromatographic separation is the most frequently techniques used in research on the infochemicals and chemical analysis of natural products. In this paper, three secondary metabolites were isolated with fat-soluble substances from the black ants, Polyrhachisvicina Roger by normalphase high-performance liquid chromatography ( NP-HPLC ) and Sephadex LH-20.And their structure were identified as 3-phenyl, 5, 6-hydride, 1,2,4-triazole (compound 1 ), $\beta$-sitosterol ( compound 2) and 24-ethyl- $\beta$-sitosterol ( compound 3 ).

\section{Introduction}

Infochemicals is a new comprehensive system disciplines, in which was used researching on drugs and the content $\&$ flow of information of drug-related system. It can greatly speed up the process of drug research, shorten the research period, reduce the research costs when introducing the infochemicals into the drug discovery process and from the whole process of drug research, almost every aspect have a close relationship with bioinformatics and chemistry ${ }^{[1]}$.

Such as natural products are the most reliable and successful source of biomaterials. A strategy to expand the range of natural products research available for the screening of usefully biomaterials for leading compounds in drug discovery. At the early study, we find that ant contains large amounts of nitrogen-containing compounds and triterpenoids ${ }^{[2-4]}$. In this research, We provide a method by chromatographic separation techniques to extract and isolated traces secondary metabolites of insects from black ants, Polyrhachis vicina Roger.

\section{Experimental}

Materials The black ants, Polyrhachisvicina Roger were collected from Luoyang Herbal biopharmaceutical AG. A voucher specimen (PLF2015) was deposited in the specimen Hall of Natural Products Chemistry, School of Chemical Engineering and Pharmaceutics, Henan University of Science and Technology.

Extract and Isolation The dry bodies of black ants, Polyrhachisvicina Roger (1.0 kg) were ground and ultrasonic percolated three times with petroleum ether (each $1 \mathrm{~L}, 48 \mathrm{~h}$ ) at room temperature followed by concentration of the extract under reduced pressure to give the oil $(140 \mathrm{~g})$. Residue of the black ants, Polyrhachis vicina Roger was dried, then percolated three times with $70 \%$ methanol (each $1 \mathrm{~L}, 48 \mathrm{~h})$ at room temperature through ultrasound auxiliary, and by concentration of the extract under reduced pressure to give the crude extract $(60 \mathrm{~g})$. Dissolve the crude extract fully with $\mathrm{H}_{2} \mathrm{O}$ then extract with EtOAc and 70\% methanol repeatedly. EtOAc layer was dried under vacuum to yield $20 \mathrm{~g}$ of crude extract, and $35 \mathrm{~g}$ of $70 \%$ methanol layer as well. Dissolve the EtOAc layer extract fully with solvent then mixing with silica gel 100-200m (Qingdao Marine Chemical Co., Inc.), remove the solvent at the fume hood. Then Column chromatography was performed using silica gel 200-300m with petroleum ether and EtOAc system, and gradient elution with petroleumether : EtOAc ( 1:0, 15:1, 10:1, 5:1, 1:1, 1:5, 1:10, 0:1 ) and get the fraction Fr.1 Fr.10. 
The Fr.3 Fr.5 ( $500 \mathrm{mg}$, eluted by petroleumether : EtOAc=15:1) perform the normal phase high-performance liquid chromatography ( NP-HPLC ) separation on a Waters-600E HPLC system with a RID detector ( RI2000, preparative capillary IN/OUT $=1.0 \mathrm{~mm}$ ID Flowcell: $7 \mu \mathrm{l}$ ). In this process, a semi-preparative column (YMC-Pack R\&D SIL 20x250mml.D.S-5 $\mu \mathrm{m}$. 12nm SL12S05-2520WYX) have been used for the separation and purification of the goal compounds, petroleumether and EtOAc $(15: 1)$ were used as NP-HPLC eluents (the mobile phase) with $5 \mathrm{ml} / \mathrm{min}$ flow rate at $20^{\circ} \mathrm{C}$. Repeated the NP-HPLC chromatography technology separation and purification of all these fractions (500mg) in the above condition, two compounds P01 (compound 2,150mg) and P03( compound 3, 200mg ) were purified at $14 \mathrm{~min}$ and $18 \mathrm{~min}$ retention time, The semi-preparative and purification of compounds 2 and 3 was shown in Figure 1 3.

The Fr.6 Fr.7 ( $80 \mathrm{mg}$, eluted by petroleumether : EtOAc $=1: 5$ ) perform the column

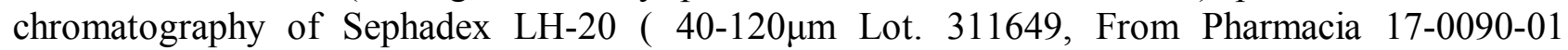
Cat.No.S8110). Chloroform and methanol ( $1: 1$ ) were used as the mobile phase eluents with $1 \mathrm{ml} / 3 \mathrm{~min}$ flow rate at $25^{\circ} \mathrm{C}$, the subfraction $(30 \mathrm{ml}$,solution ) was obtained at $150 \sim 240$ min retention time, reduce pressure concentration to give the compound P06 ( compound 1,50mg ). The purification spectrogram of compound 1 was shown in Figure 4.

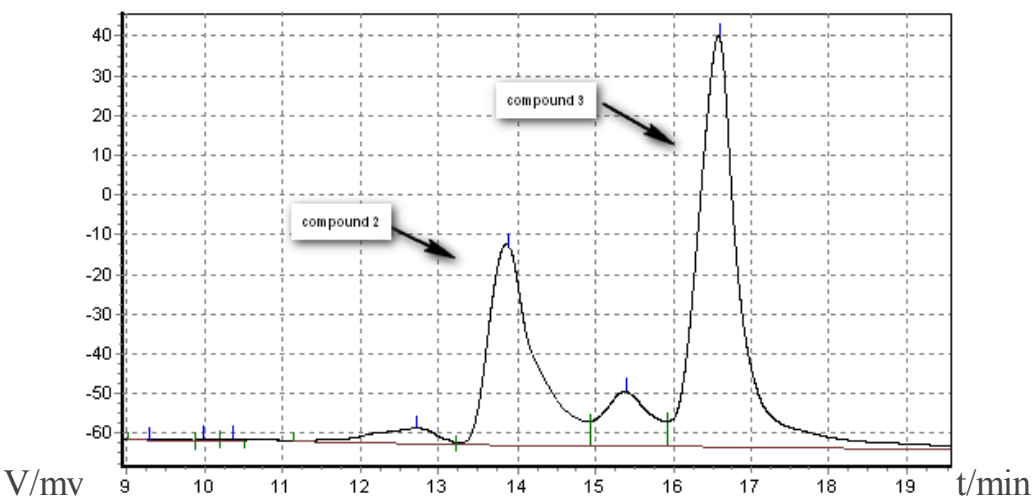

Fig.1. Isolation and purification of compound $2-3$ by NP-HPLC

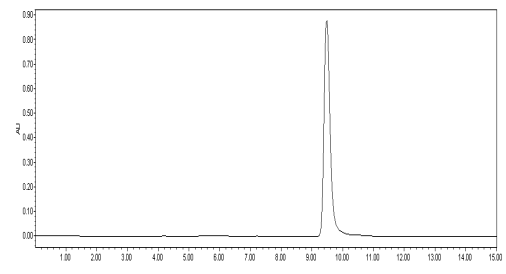

Fig.2 purefaction of compound 2

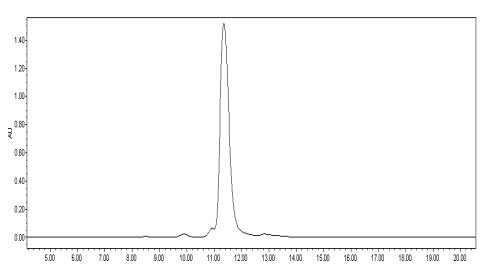

Fig. 3. purifaction of compound 3

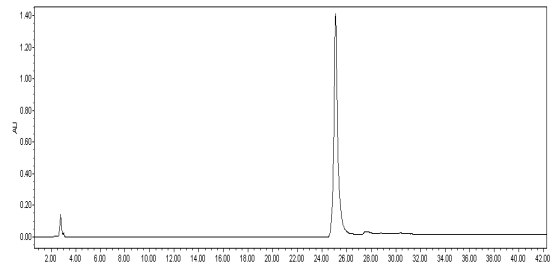

Fig. 4 purefaction of compound 1

\section{Results and Discussion}

Structure identification of the compounds isolated from the black ants, Polyrhachis vicina Roger. All the isolated as a naturally occurring compound and characterized by using ${ }^{1} \mathrm{H}$ NMR and ${ }^{13} \mathrm{C}$ NMR spectroscopy for the first time in this investigation.

Compound 1: white solid, solubility in DMSO, According to the NMR spectral data and the literature reported ${ }^{[5]}$, the compound can be identified as 3-phenyl, 5, 6 -hydride, 1, 2, 4 - triazole ( Figure 5.). 


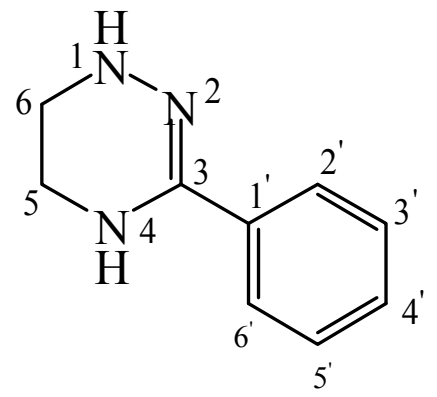

Fig. 5. Chemistry structure of compound 1.

Complete ${ }^{1} \mathrm{H}-\mathrm{NMR},{ }^{13} \mathrm{C}-\mathrm{NMR}$ chemical shift of compound 1 assignments are presented in Table $\mathbf{1 .}$

Table.1. ${ }^{1} \mathrm{H}$ NMR ( $400 \mathrm{M} \mathrm{Hz}$ ) and ${ }^{13} \mathrm{C}$ NMR ( $100 \mathrm{M} \mathrm{Hz}$ ) data assigned of compound $\mathbf{1}$

\begin{tabular}{clc} 
No. & \multicolumn{1}{c}{$\delta_{H}$} & $\delta_{C}$ \\
\hline 3 & & 166.1 \\
4 & & \\
5 & $\mathrm{H} \alpha 3.99(\mathrm{~m}, 1 \mathrm{H})$ & 55.3 \\
& $\mathrm{H} \beta 2.54(\mathrm{~d}, 4.84,1 \mathrm{H})$ & \\
6 & $\mathrm{H} \alpha 0.61(\mathrm{~m}, 1 \mathrm{H})$ & 55.3 \\
$1^{\prime}$ & $\mathrm{H} \beta 2.22(\mathrm{~d}, 4.84,1 \mathrm{H})$ & 136.4 \\
$2^{\prime}$ & $7.23(\mathrm{~m}, 1 \mathrm{H})$ & 129.7 \\
$3^{\prime}$ & $7.30(\mathrm{dt}, 2 \mathrm{H})$ & 128.1 \\
$4^{\prime}$ & $7.04(\mathrm{dt}, 2 \mathrm{H})$ & 126.4 \\
$5^{\prime}$ & $7.04(\mathrm{~m}, 1 \mathrm{H})$ & 128.1 \\
$6^{\prime}$ & $7.30(\mathrm{dt}, 2 \mathrm{H})$ & $12 \mathrm{H})$ \\
\hline
\end{tabular}

Compound 2: white solid, solubility inchloroform, mp $136 \sim 137^{\circ} \mathrm{C}$, Molishreaction was negative, Liebermann-Burchard reaction was positive.1H NMR and 13C NMR Spectra show a pair of unsaturated double signal,and six typical sterols methy peaks in high-field region. Also it has the same Rf with $\beta$-Sitosterol Standard in TLC.

The above physicochemical properties and spectral data was anastomotic with Literature ${ }^{[6]}$, so the compound can be identified as $\beta$ - Sitosterol. As shown in Figure 6.

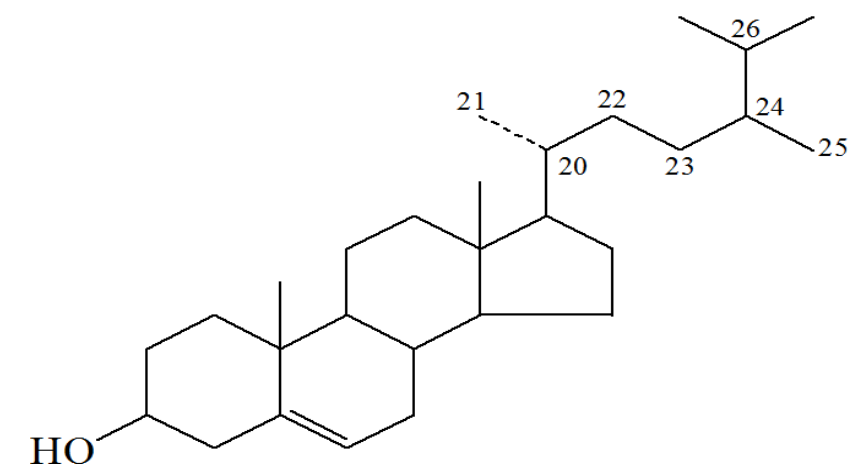

Fig. 6. Chemistry structure of compound 2.

Compound 3: white solid, solubility inchloroform, $\mathrm{mp} 136 \sim 137^{\circ} \mathrm{C}$, Molishreaction was negative, Liebermann-Burchard reaction was positive. 1H NMR and 13C NMR Spectra show it was more than two carbon peaks ( $\delta c 42.30$ and 12.06 ) when compared to $\beta$ - sitosterol, Control with document ${ }^{[7]}$, the compound is similar to ergosterol in branched-chain saturated hydrocarbon, just more than one ethyl in C-24. 
So the compound can be identified as $24-$ ethyl- $\beta$-Sitosterol. As Figure ${ }^{0} 7$.

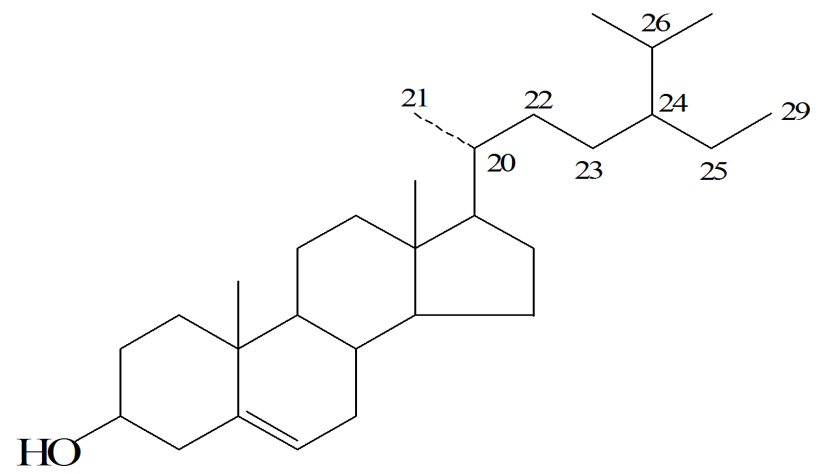

Fig 7. Chemistry structure of compound 3.

\section{Conclusions}

Due to the application of modern analysis techniques, the process of chromatography separation with chemical analysis is becoming more and more convenient and quick, especially in the chemical information from them. Among of them, Compound 1 and 3 of the secondary metabolites substances were isolated from blank ants, Polyrhachis vicina Roger for the first time.

\section{Acknowledgements}

This study was supported by National Natural Science Foundation of China (Grant No.21242011) and was also financially supported by Henan Science and Technology Foundation (020131020).

\section{References}

[1] D.S. Cao, A new method of chemistry Bioinformatics and its application in medicine research, Central South University, Master's thesis, ( in Chinese ), 2013.

[2] W. Zheng, L.J. Guo and X.Q. Tan, The Rsearch of Polyrhachisvicina Roger chemical constituents, Cent. South. Pharm. 10 (2012) 178-180.

[3] Y.X. Cheng, P. Fang and H.L. Xia, Constituents from the edible Chinese black ants (Polyrhachis dives) showing protective effect on rat mesangial cells and anti-inflammatory activity, Food Research International. 67 (2015), 163-168.

[4] Y.X. Cheng, J.J. Tang and L.P. Jiang, Dopamine derivatives from the insect Polyrhachis dives as inhibitors of ROCK1/2 and stimulators of neural stem cell proliferation, Tetrahedron. 70 (2014), 8852-8857.

[5] S.P.C. Venkata, P. Indra, Insolation of Stigmasterol and $\beta$-Sitosterol from the dichloromethane extract of Rubussuavissimus, International Current Pharmaceutical Journal. 9 (2012), 239-242.

[6] Y.H. Gong, Natural organic compounds 13C NMR chemical shift, Yunnan Science and Technology Press, Kunming (in Chinese), 1985, p.266.

[7] Y.H. Gong, Natural organic compounds 13C NMR chemical shift, Yunnan Science and Technology Press, Kunming (in Chinese), 1985, p. 51. 